\title{
Thymoquinone reduces spinal cord injury by inhibiting inflammatory response, oxidative stress and apoptosis via PPAR- $\gamma$ and PI3K/Akt pathways
}

\author{
YINMING CHEN, BENLONG WANG and HAI ZHAO \\ Department of Orthopedics, Zaozhuang Municipal Hospital, Zaozhuang, Shandong 277102, P.R. China
}

Received February 21, 2017; Accepted September 1, 2017

DOI: $10.3892 /$ etm.2018.6072

\begin{abstract}
The present study used a mild contusion injury in rat spinal cord to determine that thymoquinone reduces inflammatory response, oxidative stress and apoptosis in a spinal cord injury (SCI) rat model and to demonstrate its possible molecular mechanisms. The rats in the thymoquinone group received $30 \mathrm{mg} / \mathrm{kg}$ thymoquinone once daily by intragastric administration from 3 weeks after surgery. Hematoxylin and eosin staining, Basso, Beattie and Bresnahan (BBB) scale and tissue water content detection were used in the present study to analyze the effect of thymoquinone on SCI. The activity of inflammatory response mediators, oxidative stress factors and caspase-3/9 was measured using ELISA kits. Furthermore, western blotting was performed to analyzed the protein expression levels of prostaglandin E2, suppressed cyclooxygenase-2 $(\mathrm{COX}-2)$ and activated peroxisome proliferator-activated receptor $\gamma$ (PPAR- $\gamma$ ), PI3K and Akt. The results from the study demonstrated that thymoquinone increased Basso, Beattie and Bresnahan score and decreased water content in spinal cord tissue. Treatment with thymoquinone decreased inflammatory response [measured by levels of tumor necrosis factor $\alpha$, interleukin (IL)-1 $\beta$, IL-6 and IL-18], oxidative stress (measured by levels of superoxide dismutase, catalase, glutathione and malondialdehyde) and cell apoptosis (measured by levels of caspase-3 and caspase-9) in SCI rats. Thymoquinone treatment inhibited prostaglandin E2 activity, suppressed COX-2 protein expression and activated PPAR- $\gamma$, PI3K and $\mathrm{p}-\mathrm{Akt}$ protein expression in SCI rats. These data revealed that thymoquinone reduces inflammatory response, oxidative stress and apoptosis via PPAR- $\gamma$ and PI3K/Akt pathways in an SCI rat model.
\end{abstract}

Correspondence to: Dr Benlong Wang, Department of Orthopedics, Zaozhuang Municipal Hospital, 41 Longtou Middle Road, Zaozhuang, Shandong 277102, P.R. China

E-mail: wangbenlongsd@163.com

Key words: thymoquinone, spinal cord injury, peroxisome proliferator-activated receptor $\gamma$, phosphoinositide 3-kinase/Akt

\section{Introduction}

Acute spinal cord injury (SCI) is a serious nervous system injury, which often results in partial or complete loss of feeling and motor function below the injury surface. According to a previous report, the annual incidence of SCI is $15-40 / 1,000,000$ worldwide (1). SCI can be caused by traffic accidents, falls or sports injuries. Of these, traffic accidents are the main cause (44.5\%) of SCI in the USA, followed by falls (16.6\%) and sports injuries (12.7\%) (1). The injured people are commonly of working age (range, 18-80 years old). Research has indicated that SCI has brought great economic burden on individuals, families and society (2). Therefore, treatment for acute SCI, including nerve damage recovery or reduction, has great social significance. Nonetheless, SCI treatment remains a challenge in the medical field.

Acute SCI can be classified into primary SCI and secondary SCI. Secondary SCI was proposed by Allen in 1911 (3). Primary SCI refers to mechanical injury to part of the spinal cord. The magnitude of primary injury is determined by the external force at the moment of impact. Such injury is irreversible, so it is not an effective treatment strategy (4). Secondary injury refers to serious damage within a few minutes of the primary injury, including edema, inflammation, ischemia, excessively activated glutamate receptors, lipid peroxidation and calcium overload. This will result in secondary cell death for a time period ranging from several days to weeks. Thus, it will cause massive death of neurons and glia cells after primary injury (5). The severity of multifactorial sequence tissue destruction is even greater than that of primary injury. The injury area will develop serial reactions after SCI. It will not only damage residual nerve cells, but also cause damage the spinal cord tissue surrounding the injury center (6).

Previous results have verified that inflammation aggravates the post-SCI secondary injury (7). Apoptosis is the key manifestation of secondary injury; thus, inhibition of apoptosis can prevent or reduce secondary injury, protect nerve function and alleviate nerve cell loss (7). SCI has allowed the surviving cells to survive in primary injury and it also allows more nerve function to be retained (8). Apoptosis, which is also known as programmed cell death, is an active death process under the regulation of multiple signaling pathways (9). Energy consumption is required during cell apoptosis, so as to synthesize new proteins and nucleic acids (10). 
Peroxisome proliferator-activated receptor (PPAR) is a member of the ligand activated nuclear transcription factor superfamily (11). PPAR agonists have been demonstrated in previous studies to exhibit anti-inflammatory effects and inhibitory effects on brain neural apoptosis $(11,12)$. Research on PPAR- $\gamma$ in SCI has focused little on its effects of protecting spinal neurons and promoting post-SCI secretion of inflammatory cytokines (12). The current study was thereby conducted aiming to observe the effects of PPAR- $\gamma$ agonist thymoquinone on repairing SCI in rats. Furthermore, its mechanism was investigated in order to provide therapeutic strategies for clinical SCI (13).

SCI treatments will promote nerve growth factor (NGF), activating the phosphoinositide 3-kinase (PI3K)/Akt pathway to inhibit neuron apoptosis (14). Therefore, post-SCI, it is of particular importance to adapt proper methods to induce NGF activation and proliferation, repair the damaged spinal cord nerve and function of bladder, and activate the PI3K/Akt pathway to suppress apoptosis, thus improving neural function (15).

Black cumin, Nigella sativa, belongs to the family Ranunculaceae, and is an annual herbaceous plant. It is used as a traditional natural medicine for numerous diseases (16). Thymoquinone (Fig. 1) is the primary active ingredient of black cumin seed oil and is commonly used for anti-inflammation, anti-oxidation and anti-tumor treatment (17). Over the past decade, thymoquinone could inhibit numerous cancer types, including breast, prostate, ovarian, liver, pancreatic and colorectal cancer (18-20). The present study aimed to determine the effect of thymoquinone on inflammatory response, oxidative stress and apoptosis in SCI rats, and to investigate its possible molecular mechanisms.

\section{Materials and methods}

Animals. Male Sprague Dawley rats (age, 6 weeks; weight, $180 \pm 10 \mathrm{~g}, \mathrm{n}=26$ ) were purchased from the Animal Experiment Center of Shandong University (Shandong, China) and individually housed (temperature, $23 \pm 1^{\circ} \mathrm{C} ; 55-60 \%$ humidity) and were exposed to a $12 \mathrm{~h}$ light/dark cycle (lights on from 8:00 a.m. to 8:00 p.m.). Rats also had free access to food and water ad libitum. This study was performed in accordance with the guidelines of the National Institutes of Health of Zaozhuang Municipal Hospital as referred to previously (21), and approved by Zaozhuang Municipal Hospital of Care and Use Committee.

Surgical procedures and experimental setup. The rats were randomly divided into three groups: Sham surgery (sham, $\mathrm{n}=6$ ), SCI surgery (model, $\mathrm{n}=10$ ) and SCI + thymoquinone (thymoquinone, $\mathrm{n}=10$ ). The rats from the model and thymoquinone groups were anesthetized with $400 \mathrm{mg} / \mathrm{kg}$ of chloral hydrate, and a laminectomy was performed at the T9-T10 level. The underlying cord was exposed to contusion injury without disrupting the dura (22). The thymoquinone group received thymoquinone at $30 \mathrm{mg} / \mathrm{kg}$ once daily by intragastric administration (23) from 3 weeks after surgery. The rats from the sham and model groups received an equal volume of vehicle (PBS) at the same time. In the sham group, rats were anesthetized with $400 \mathrm{mg} / \mathrm{kg}$ of chloral hydrate, and the surgical procedure was

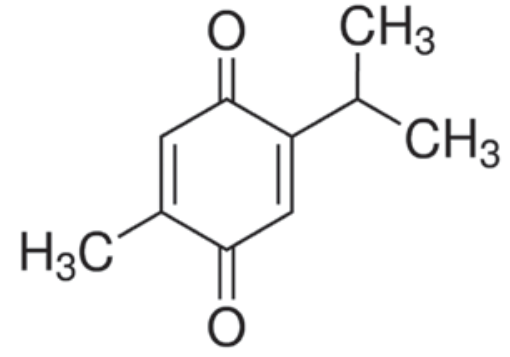

Figure 1. Structural formula of thymoquinone.

not performed. Furthermore, Sham rats received normal saline by intragastric administration for 3 weeks.

Histological assessment. Rats were anesthetized using $35 \mathrm{mg} / \mathrm{kg}$ pentobarbital sodium and then was sacrificed using decollation. Spinal cord tissue was extracted, washed with PBS and fixed in $10 \%$ neutral buffered formalin for 3 days at room temperature. Then, tissue was decalcified in 10\% EDTA for 10 days and embedded into paraffin. Next, tissue was cut into serial paraffin sections (4 mm), which were stained with hematoxylin and eosin for $30 \mathrm{~min}$ at room temperature and observed using a microscope (Olympus IX81; Olympus Corporation, Tokyo, Japan).

Behavioral assessments. The Basso, Beattie and Bresnahan (BBB) scale and water content in spinal cord tissue were used to assess neurological function after treatment with thymoquinone. The BBB score is on a scale from 1 to 21 , indicating no hindlimb movement to normal hindlimb function (24). Spinal cord tissue samples were extracted after treatment with thymoquinone and washed with PBS. Tissue samples were weighed as wet weight and then dried at $72^{\circ} \mathrm{C}$ for $48 \mathrm{~h}$. Next, tissue samples were weighed as dry weight. Water content was calculated as follows: Water content $(\%)=$ wet weight $/$ dry weight $\times 100 \%$.

Measurement of inflammatory response, oxidative stress and cell apoptosis. Tumor necrosis factor (TNF)- $\alpha$ (cat. no. PT516; Beyotime Institute of Biotechnology, Haimen, China), interleukin (IL)-1 $\beta$ (cat. no. PI303; Beyotime Institute of Biotechnology), IL-6 (cat. no. PI328; Beyotime Institute of Biotechnology), IL-18 (cat. no. E-EL-R0567c; Elabscience, Houston, TX, USA), superoxide dismutase (SOD; cat. no. S0109; Beyotime Institute of Biotechnology), catalase (CAT; cat. no. S0051; Beyotime Institute of Biotechnology), glutathione (GSH; cat. no. S0052; Beyotime Institute of Biotechnology), PGE2 and malondialdehyde (MDA; cat. no. S0131; Beyotime Institute of Biotechnology), caspase-3 (cat. no. C1116; Beyotime Institute of Biotechnology) and caspase-9 (cat. no. C1158; Beyotime Institute of Biotechnology) activity levels in the spinal tissue were evaluated using ELISA kits after treatment with thymoquinone. Absorbency changes were measured using spectrophotometry at a wavelength of $450 \mathrm{~nm}$ (BioTek ELx800 Absorbance Microplate Reader; BioTek Instruments, Inc., Winooski, VT, USA). Experiments were replicated 6 times.

Western blotting. Spinal cord tissue extracts were extracted after treatment with thymoquinone, homogenized with radioimmunoprecipitation assay lysis buffer (Beyotime Institute of 


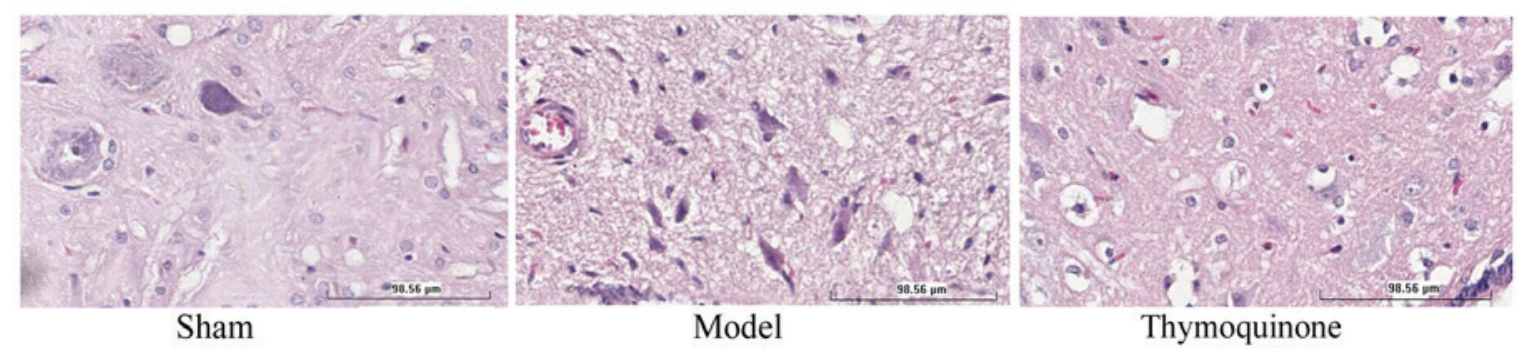

Figure 2. Effect of thymoquinone on SCI. SCI, spinal cord injury; Sham, sham surgery group; Model, SCI surgery group; Thymoquinone, SCI + thymoquinone group.
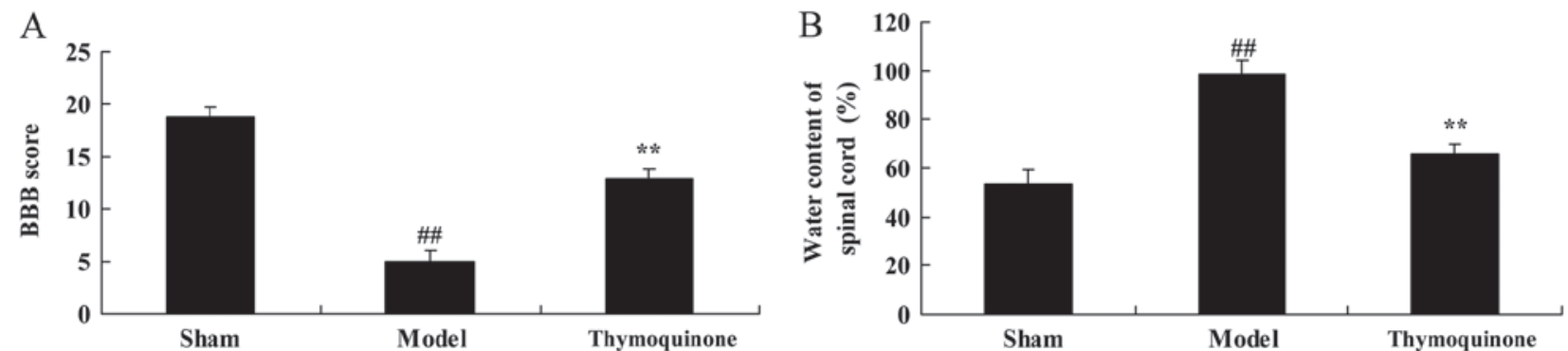

Figure 3. Effect of thymoquinone on (A) BBB score and (B) water content in spinal cord tissue. ${ }^{\# \#} \mathrm{P}<0.01 \mathrm{vs}$. Sham; ${ }^{* *} \mathrm{P}<0.01$ vs. Model. SCI, spinal cord injury; Sham, sham surgery group; Model, SCI surgery group; Thymoquinone, SCI + thymoquinone group; BBB, Basso, Beattie and Bresnahan.

Biotechnology) or $30 \mathrm{~min}$ at $4^{\circ} \mathrm{C}$, then centrifuged at $12,000 \mathrm{xg}$ for $5 \mathrm{~min}$ at $4^{\circ} \mathrm{C}$. Protein content was measured using a colorimetric protein assay kit (Bio-Rad Laboratories, Inc., Hercules, CA, USA). Protein (50 $\mu \mathrm{g}$ per sample) were loaded onto $12 \%$ polyacrylamide gels and separated by SDS-PAGE, then transferred from the gels to a nitrocellulose membrane. The membrane was blocked with $5 \%(\mathrm{w} / \mathrm{v})$ non-fat milk in Tris-buffered saline containing $0.05 \%$ Tween-20 at $37^{\circ} \mathrm{C}$ for $1 \mathrm{~h}$ and incubated with anti-cyclooxygenase 2 (COX-2; cat. no. sc-7951, dilution 1:1,000; Santa Cruz Biotechnology, Inc., Dallas, TX, USA), anti-PPAR- $\gamma$ (cat. no. sc-9000, dilution 1:1,000; Santa Cruz Biotechnology, Inc.), anti-PI3K (cat. no. sc-7175, dilution 1:1,000; Santa Cruz Biotechnology, Inc.), anti-Akt (cat. no. sc-8312, dilution 1:500; Santa Cruz Biotechnology, Inc.), anti-p-Akt (cat. no. sc-7985-R, dilution 1:1,000; Santa Cruz Biotechnology, Inc.) and anti-GAPDH (cat. no. sc-25778, dilution 1:2,000; Santa Cruz Biotechnology, Inc.) at $4^{\circ} \mathrm{C}$ overnight. The membrane was incubated with horseradish peroxidase-conjugated goat anti-rabbit secondary antibody (dilution 1:5,000, cat. no. sc-2004; Santa Cruz Biotechnology, Inc.) for $1 \mathrm{~h}$ at $37^{\circ} \mathrm{C}$ and visualized with an enhanced chemiluminescence system using sodium Image_Lab_3.0 (Bio-Rad Laboratories, Inc.). Experiments were replicated three times.

Statistical analysis. Data are expressed as the mean \pm standard deviation using SPSS 17.0 (SPSS, Inc., Chicago, IL, USA). Statistical differences were determined using one-way ANOVA followed by Tukey's test. $\mathrm{P}<0.05$ was considered to indicate a statistically significant difference.

\section{Results}

Thymoquinone reduces symptoms of SCI. To investigate the in vivo effects of thymoquinone on SCI, SCI rats were treated with thymoquinone from 3 weeks after surgery. SCI model promoted necrosis in the SCI model group compared with the sham group (Fig. 2). Furthermore, the administration of thymoquinone reduced necrosis in SCI rats as compared with the SCI model group (Fig. 2).

Thymoquinone increases $B B B$ score and reduces water content in spinal cord tissue. Next, BBB score and water content in spinal cord tissue were analyzed in SCI rats treated with thymoquinone. As shown in Fig. 3A, a significant decrease in BBB score was observed in the SCI model group compared with the sham group $(\mathrm{P}<0.01)$. As shown in Fig. 3B, a significant increase of water content was observed in the SCI model group tissue, compared with the sham group $(\mathrm{P}<0.01)$. Treatment with thymoquinone significantly increased BBB score and significantly reduced spinal cord tissue water content in SCI rats as compared with the SCI model $(\mathrm{P}<0.01$; Fig. 3A and B). These results demonstrated that thymoquinone could prevent SCI, but its mechanism required further elucidation.

Thymoquinone decreases inflammatory responses in SCI rats. The levels of inflammatory factors were analyzed to determine whether thymoquinone affected the inflammatory response in SCI rats. As shown in Fig. 4, TNF- $\alpha$, IL-1 $\beta$, IL-6 and IL-18 activity levels in the SCI model group were significantly higher compared with the sham group $(\mathrm{P}<0.01)$. However, TNF- $\alpha$, IL-1 $\beta$, IL- 6 and IL-18 activity levels were significantly decreased in SCI rats treated with thymoquinone compared with the SCI model group (Fig. 4). These results indicated that thymoquinone exhibits anti-inflammatory effects in the treatment of SCI.

Thymoquinone decreases oxidative stress in SCI rats. In order to determine whether thymoquinone affects oxidative stress 

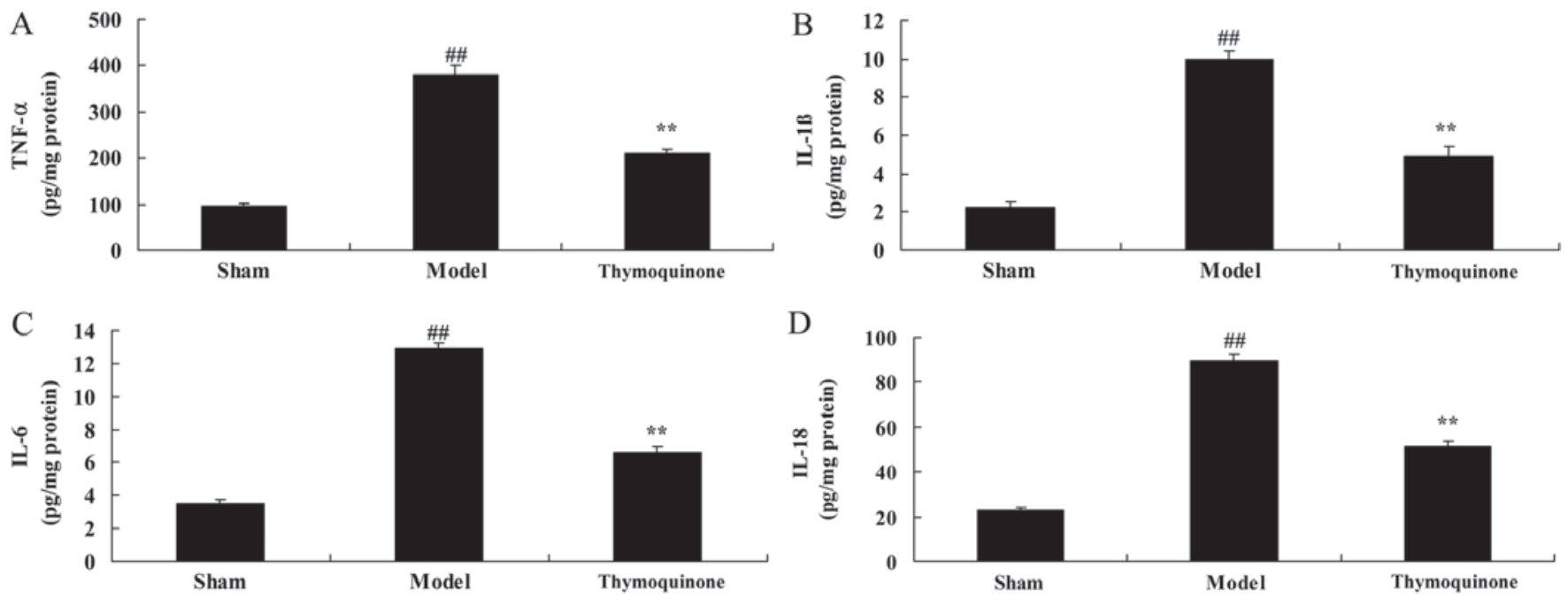

Figure 4. Effect of thymoquinoneon inflammatory responses in SCI rats. Activity levels of (A) TNF- $\alpha$, (B) IL-1 $\beta$, (C) IL-6 and (D) IL-18 were measured using ELISA kits. ${ }^{\# \#} \mathrm{P}<0.01$ vs. Sham; ${ }^{* *} \mathrm{P}<0.01$ vs. Model. SCI, spinal cord injury; Sham, sham surgery group; Model, SCI surgery group; Thymoquinone, SCI + thymoquinone group; TNF, tumor necrosis factor; IL, interleukin.
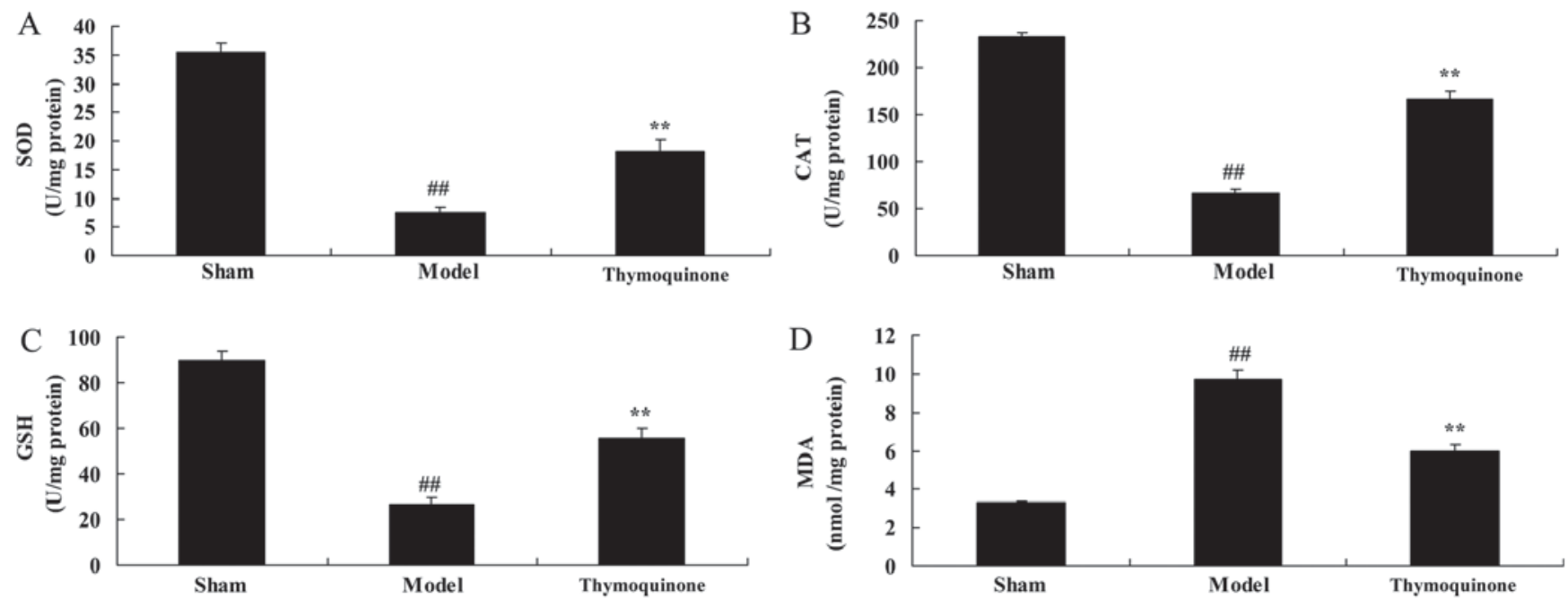

Figure 5. Effect of thymoquinone on oxidative stress in SCI rats. Activity levels of (A) SOD, (B) CAT, (C) GSH and (D) MDA were measured using ELISA kits. ${ }^{\# /} \mathrm{P}<0.01$ vs. Sham; ${ }^{* *} \mathrm{P}<0.01$ vs. Model. SCI, spinal cord injury; Sham, sham surgery group; Model, SCI surgery group; Thymoquinone, SCI + thymoquinone group; SOD, superoxide dismutase; CAT, catalase; GSH, glutathione; MDA, malondialdehyde.

in SCI rats, SOD, CAT, GSH and MDA activity levels were measured using ELISA kits. As shown in Fig. 5, SOD, CAT and GSH levels were significantly decreased and MDA levels were significantly increased in SCI model rats compared with the sham group $(\mathrm{P}<0.01)$. Thymoquinone treatment significantly increased the SOD, CAT and GSH activity levels and significantly decreased the MDA activity level in SCI rats compared with the model group $(\mathrm{P}<0.01)$. These results indicated that thymoquinone inhibits SCI-induced oxidative stress.

Thymoquinone decreases cell apoptosis in SCI rats. In order to determine whether thymoquinone regulates cell apoptosis in SCI rats, caspase-3 and -9 activity levels were measured using ELISA kits. As shown in Fig. 6, caspase-3 and -9 activity levels were significantly higher in SCI model rats compared with the sham group $(\mathrm{P}<0.01)$. Treatment with thymoquinone significantly decreased caspase-3 and -9 activity levels in
SCI rats as compared with the SCI model $(\mathrm{P}<0.01)$. These results indicated that thymoquinone inhibits apoptosis in the treatment of SCI.

Thymoquinone inhibits prostaglandin E2 (PGE2) activity in $S C I$ rat. To characterize the mechanism of thymoquinone on SCI, PGE2 activity was analyzed in SCI rats. A significant increase in PGE2 activity was observed in SCI model rats compared with the sham group $(\mathrm{P}<0.01 ;$ Fig. 7$)$. Administration with thymoquinone significantly inhibited PGE2 activity in SCI rats as compared with the SCI model ( $\mathrm{P}<0.01$; Fig. 7 ). These results indicated that thymoquinone reduces PGE2 activity to inhibit inflammation in SCI.

Thymoquinone suppresses COX-2 and activates PPAR- $\gamma$ protein expression in SCI rats. To further characterize the mechanism of thymoquinone in SCI, COX-2 and PPAR- $\gamma$ 

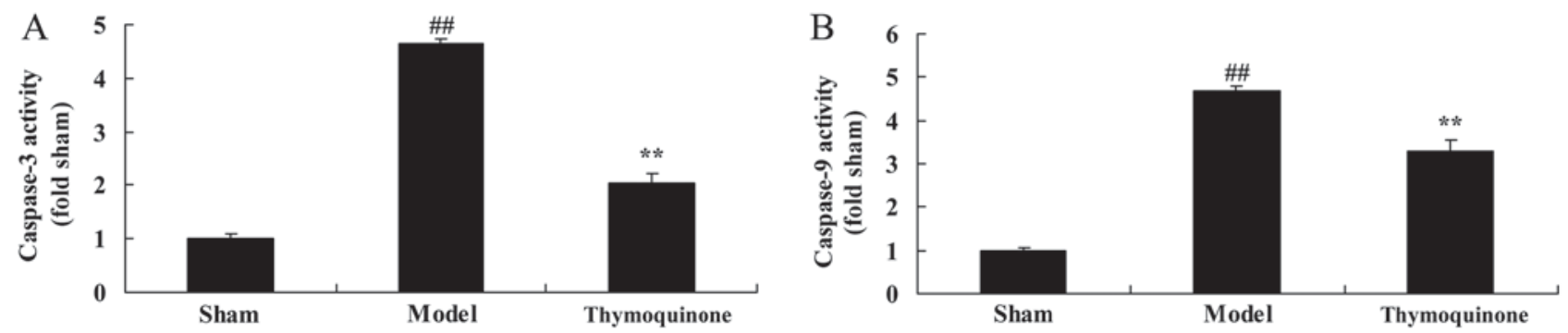

Figure 6. Effect of thymoquinone on cell apoptosis in SCI rats. Activity levels of (A) caspase-3 and (B) caspase-9 were measured using ELISA kits. ${ }^{\sharp \#} \mathrm{P}<0.01$ vs. Sham; ${ }^{* *} \mathrm{P}<0.01$ vs. Model. SCI, spinal cord injury; Sham, sham surgery group; Model, SCI surgery + exercise group; Thymoquinone, $\mathrm{SCI}+$ thymoquinone group.

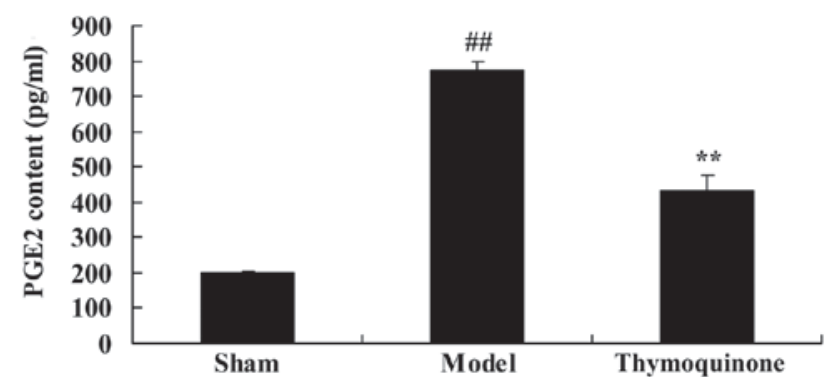

Figure 7. Effect of thymoquinone on PGE2 activity in SCI rats. ${ }^{\# \#} \mathrm{P}<0.01$ vs. Sham; ${ }^{* *} \mathrm{P}<0.01$ vs. Model. SCI, spinal cord injury; Sham, sham surgery group; Model, SCI surgery group; Thymoquinone, SCI + thymoquinone group; PGE2, prostaglandin E2.

protein expression were analyzed. As shown in Fig. 8, COX-2 protein expression was significantly increased and PPAR- $\gamma$ protein expression was significantly decreased in SCI model rats as compared with the sham group $(\mathrm{P}<0.01)$. Thymoquinone significantly suppressed COX-2 and increased PPAR- $\gamma$ protein expression in SCI rats as compared with the SCI model rats $(\mathrm{P}<0.01$; Fig. 8). These results indicated that thymoquinone reduces COX-2 activity to inhibit inflammation, and increases PPAR- $\gamma$ to reduce apoptosis in SCI.

Thymoquinone activates PI3K and $p$-Akt/Akt protein expression in SCI rats. In order to evaluate whether the PI3K/Akt pathway was downregulated early after SCI and whether thymoquinone could regulate the PI3K/Akt pathway, PI3K and p-Akt/Akt protein expression were analyzed. There was a significant suppression of PI3K and p-Akt/Akt protein expressionin the SCI model group compared with the sham group $(\mathrm{P}<0.01$; Fig. 9). Notably, thymoquinone treatment significantly promoted the PI3K and p-Akt/Akt protein expression in SCI rats as compared with the SCI model $(\mathrm{P}<0.01$; Fig. 9). These results suggest that the PI3K/Akt pathway is involved in the effects of thymoquinone in SCI.

\section{Discussion}

SCI is a serious nervous system injury, which can cause motor dysfunction and severe disability, thus causing a large economic burden to individuals, families and society (25). Therefore, it is of great significance to treat acute SCI and recover or alleviate nerve damage. Acute SCI is currently classified into primary and secondary SCI (26). Secondary SCI is reversible and can be controlled. Consequently, secondary SCI determines the patient's final outcome (27). At present, treatment of secondary SCI is the key strategy for acute SCI treatment. Inflammatory response is the primary component of secondary injury of the spinal cord (25). It was identified in the present study that thymoquinone can reduce SCI, increase BBB score and decrease water content in spinal cord tissue in SCI rat.

Cytokines, inflammation, free radicals, excitatory toxins and other factors can trigger apoptosis (28). Researchers have identified that apoptosis associated genes are involved. For instance, genes including caspase-3, B-cell lymphoma 2 (Bcl-2) and $\mathrm{Bcl}-2$-associated $\mathrm{X}$ protein are associated with apoptosis (29). In particular, caspase-3 is closely correlated with apoptosis regulation (29). In addition, caspase-3 activity has formed the positive and negative regulation of apoptosis, while the ratio between the two decides cell apoptosis $(29,30)$. Massive neuron apoptosis has been identified in numerous central nervous system injury models (31). The present data indicate that thymoquinone can decrease inflammatory response, oxidative stress and cell apoptosis in an SCI rat model. Dur et al (17) demonstrated that thymoquinone could prevent inflammation and oxidative stress in rat acute pancreatitis. These results suggest that thymoquinone exerts an anti-inflammatory, anti-oxidative and anti-apoptotic effect on SCI.

Previous studies on PPAR $-\gamma$ have primarily focused on lipid metabolism and internal environment stability. It has been identified that PPAR- $\gamma$ is involved in numerous physiological and pathological processes (32). In addition, PPAR- $\gamma$ agonists have been demonstrated to reduce marked neuron loss after being injected into the cerebral cortex (33). During pretreatment of neural and glial cells, upregulated PPAR- $\gamma$ expression can upregulate GLT1/EAAT mRNA expression, which can also be observed in PPAR- $\gamma$ agonist-cultured cells. Thus, a nerve protective effect can be achieved (34). PPAR- $\gamma$ activation contributes to reducing the injury effect of free radicals on nerves through multiple pathways. It has been demonstrated that PPAR- $\gamma$ activation can inhibit the expression of free radicals in patients with progressive spinal muscular atrophy, multiple sclerosis and inflammation of ischemia-based nervous system (11). In inflammatory responses, PPAR- $\gamma$ can inhibit related inflammatory signal pathways in a competitive manner for the formation of inflammatory mediators (33). The current results suggest that thymoquinone treatment can inhibit PGE2 activity, suppress COX-2 protein expression and promote PPAR- $\gamma$ protein expression in SCI rats. In addition, Pei et al (35) demonstrated that thymoquinone inhibited angiotens in II-induced vascular smooth muscle cell proliferation 


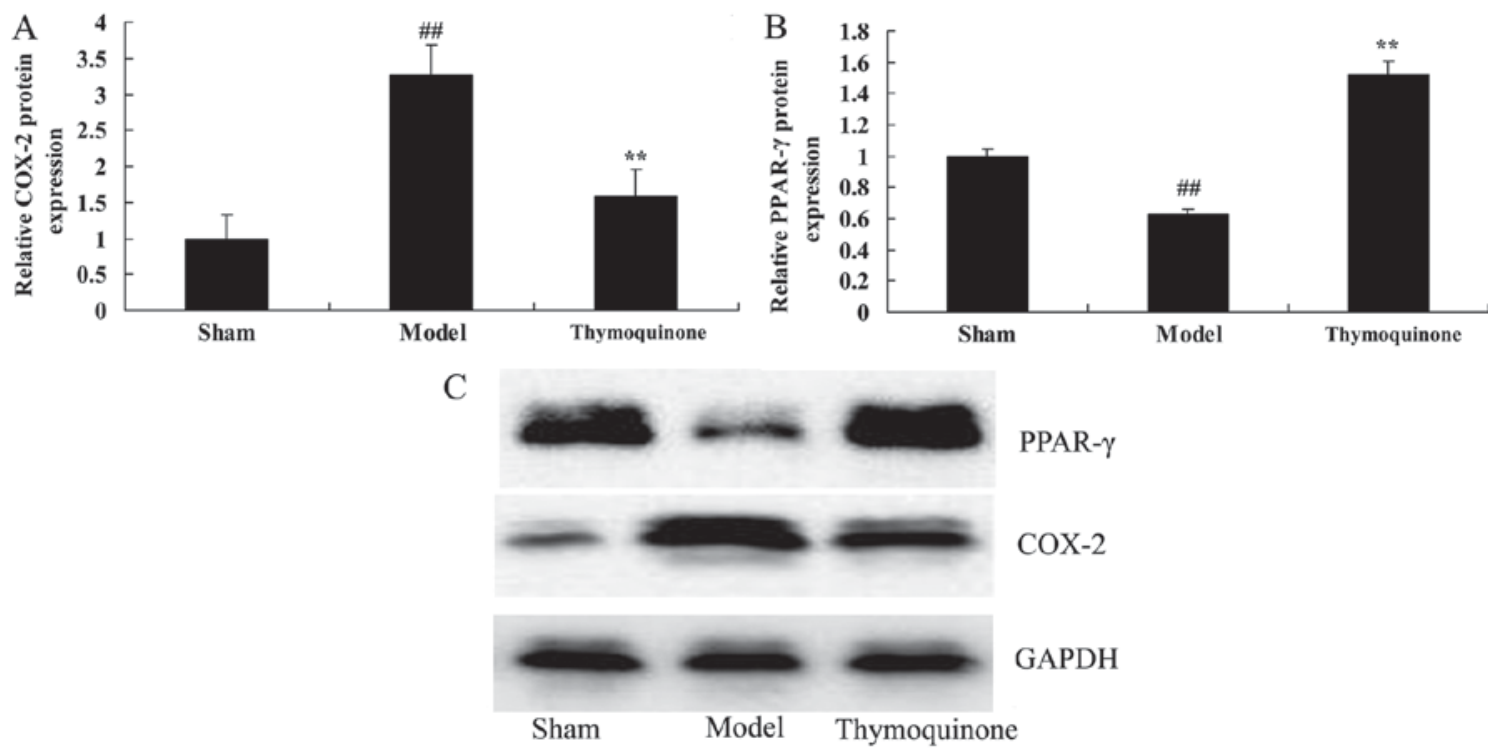

Figure 8. Effect of thymoquinoneon COX-2 and PPAR- $\gamma$ protein expressionin SCI rats. (A) COX-2 and (B) PPAR- $\gamma$ protein expression were analyzed by (C) western blotting in SCI rats. ${ }^{\# \#} \mathrm{P}<0.01$ vs. Sham; ${ }^{* *} \mathrm{P}<0.01$ vs. Model. SCI, spinal cord injury; Sham, sham surgery group; Model, SCI surgery group; Thymoquinone, SCI + thymoquinone group; COX, cyclooxygenase; PPAR, peroxisome proliferator-activated receptor.
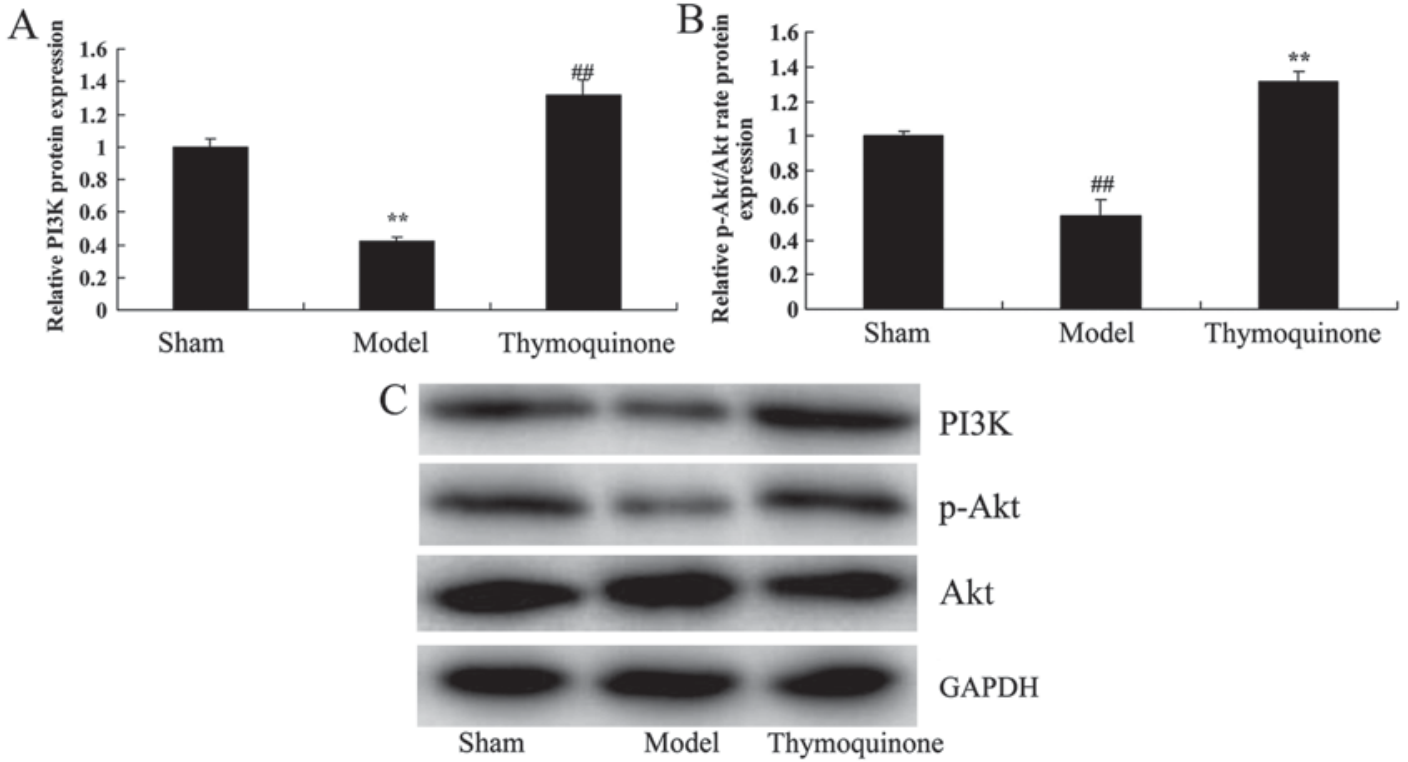

Figure 9. Effect of thymoquinone on PI3K/Akt protein expression in SCI rats. (A and B) Relative PI3K and p-Akt/Akt protein expression was analyzed by (C) western blotting in SCI rats. ${ }^{\# \#} \mathrm{P}<0.01$ vs. Sham; ${ }^{* *} \mathrm{P}<0.01$ vs. Model. SCI, spinal cord injury; Sham, sham surgery group; Model, SCI surgery group; Thymoquinone, $\mathrm{SCI}+$ thymoquinone group; PI3K, phosphoinositide 3-kinase.

through the PPAR- $\gamma /$ PPAR- $\gamma$ coactivator-1 pathway. These results suggested that treadmill exercise could promote the protective effect of thymoquinone on SCI by stimulating the expression of PPAR- $\gamma$.

The PI3K/Akt signaling pathway responds to extracellular signals, growth factors and the energy status of the cell, as well as cell growth, proliferation, survival and differentiation of SCI (36). This pathway serves a key function in neural physiological and pathological processes (36). As previously demonstrated, the PI3K/Akt pathway is vital in neural cell proliferation, development, differentiation, axonal regeneration, myelin formation, apoptosis and plasticity of synapses $(37,38)$. The results of the current study demonstrate that thymoquinone treatment contributes to activating PPAR- $\gamma$ and PI3K/Akt protein expression in SCI rats. Liu et al (39) suggested that thymoquinone improves cardiovascular function, and inhibits inflammation, oxidative stress and apoptosis via the PI3K/Akt pathway in diabetic rats. These results suggest that thymoquinone induces activation of the PI3K/Akt signaling pathway, which may be associated with its protective effect against SCI.

The present study demonstrated that thymoquinone can reduce SCI, increase BBB score and decrease water content in the spinal cord tissue of an SCI rat model. The protective 
effects of thymoquinone on SCI may be attributed to its activation of PPAR $-\gamma$ and the PI3K/Akt pathway.

\section{Acknowledgements}

Not applicable.

\section{Funding}

No funding was received.

\section{Availability of data and materials}

The datasets used and/or analyzed during the current study are available from the corresponding author on reasonable request.

\section{Authors' contributions}

$\mathrm{YC}$ and $\mathrm{BW}$ conceived and designed the experiments. $\mathrm{YC}$ and $\mathrm{HZ}$ performed the experiments. $\mathrm{YC}$ and $\mathrm{BW}$ analyzed the data and wrote paper.

\section{Ethics approval and consent to participate}

Not applicable.

\section{Consent for publication}

Not applicable.

\section{Competing interests}

The authors declare that they have no competing interests.

\section{References}

1. Arora M, Harvey LA, Hayes AJ, Chhabra HS, Glinsky JV, Cameron ID, Lavrencic L, Arumugam N, Hossain S and Bedi PK: Effectiveness and cost-effectiveness of telephone-based support versus usual care for treatment of pressure ulcers in people with spinal cord injury in low-income and middle-income countries: Study protocol for a 12-week randomised controlled trial. BMJ Open 5: e008369, 2015.

2. Dudley-Javoroski S and Shields RK: Active-resisted stance modulates regional bone mineral density in humans with spinal cord injury. J Spinal Cord Med 36: 191-199, 2013.

3. Harper LA, Coleman JA, Perrin PB, Olivera SL, Perdomo JL, Arango JA, and Arango-Lasprilla JC: Comparison of mental health between individuals with spinal cord injury and able-bodied controls in Neiva, Colombia. J Rehabil Res Dev 51: 127-136, 2014

4. López-Larraz E, Antelis JM, Montesano L, Gil-Agudo A and Minguez J: Continuous decoding of motor attempt and motor imagery from EEG activity in spinal cord injury patients. Conf Proc IEEE Eng Med Biol Soc 2012: 1798-1801, 2012.

5. Jia C, Liao LM, Chen G and Sui Y: Detrusor botulinum toxin A injection significantly decreased urinary tract infection in patients with traumatic spinal cord injury. Spinal Cord 51: 487-490, 2013

6. Laubacher M, Perret $\mathrm{C}$ and Hunt KJ: Work-rate-guided exercise testing in patients with incomplete spinal cord injury using a robotics-assisted tilt-table. Disabil Rehabil Assist Technol 10: 433-438, 2015

7. Rosety-Rodriguez M, Camacho A, Rosety I, Fornieles G, Rosety MA, Diaz AJ, Bernardi M, Rosety M and Ordonez FJ: Low-grade systemic inflammation and leptin levels were improved by arm cranking exercise in adults with chronic spinal cord injury. Arch Phys Med Rehabil 95: 297-302, 2014.
8. Nelissen S, Vangansewinkel T, Geurts N, Geboes L, Lemmens E, Vidal PM, Lemmens S, Willems L, Boato F, Dooley D, et al: Mast cells protect from post-traumatic spinal cord damage in mice by degrading inflammation-associated cytokines via mouse mast cell protease 4. Neurobiol Dis 62: 260-272, 2014

9. Amin B, Abnous K, Motamedshariaty V and Hosseinzadeh $\mathrm{H}$ : Attenuation of oxidative stress, inflammation and apoptosis by ethanolic and aqueous extracts of Crocus sativus L. stigma after chronic constriction injury of rats. An Acad Bras Cienc 86: 1821-1832, 2014.

10. Yu WR and Fehlings MG: Fas/FasL-mediated apoptosis and inflammation are key features of acute human spinal cord injury: Implications for translational, clinical application. Acta Neuropathol 122: 747-761, 2011.

11. Yi JH, Park SW, Brooks N, Lang BT and Vemuganti R: PPARgamma agonist rosiglitazone is neuroprotective after traumatic brain injury via anti-inflammatory and anti-oxidative mechanisms. Brain Res 1244: 164-172, 2008.

12. McTigue DM: Potential therapeutic targets for PPARgamma after spinal cord injury. PPAR Res 2008: 517162, 2008.

13. Yan J, Li B, Chen JW, Jiang SD and Jiang LS: Spinal cord injury causes bone loss through peroxisome proliferator-activated receptor- $\gamma$ and Wnt signalling. J Cell Mol Med 16: 2968-2977, 2012.

14. Chae CH and Kim HT: Forced, moderate-intensity treadmill exercise suppresses apoptosis by increasing the level of NGF and stimulating phosphatidylinositol 3-kinase signaling in the hippocampus of induced aging rats. Neurochem Int 55: 208-213, 2009.

15. Kim Y, Seger R, Suresh Babu CV, Hwang SY and Yoo YS: A positive role of the PI3-K/Akt signaling pathway in PC12 cell differentiation. Mol Cells 18: 353-359, 2004.

16. Randhawa MA, Alenazy AK, Alrowaili MG and Basha J: An active principle of Nigella sativa L., thymoquinone, showing significant antimicrobial activity against anaerobic bacteria. J Intercult Ethnopharmacol 6: 97-101,2016.

17. Dur A, Kose H, Kocyigit A, Kocaman O, Ismayilova M and Sonmez FC: The anti-inflammatory and antioxidant effects of thymoquinone on ceruleine induced acute pancreatitis in rats. Bratisl Lek Listy 117: 614-618, 2016.

18. Zhang L, Bai Y and Yang Y: Thymoquinone chemosensitizes colon cancer cells through inhibition of NF- $\kappa B$. Oncol Lett 12: 2840-2845, 2016.

19. Liu X, Dong J, Cai W, Pan Y, Li R and Li B: The effect of thymoquinone on apoptosis of SK-OV-3 ovarian cancer cell by regulation of Bcl-2 and Bax. Int J Gynecol Cancer 27: 1596-1601, 2017.

20. Barkat MA, Abul H, Ahmad J, Khan MA, Beg S and Ahmad FJ: Insights into the targeting potential of thymoquinone for therapeutic intervention against triple-negative breast cancer. Curr Drug Targets 19: 70-80, 2018.

21. Jiang ZS, Pu ZC and Hao ZH: Carvacrol protects against spinal cord injury in rats via suppressing oxidative stress and the endothelial nitric oxide synthase pathway. Mol Med Rep 12: 5349-5354, 2015.

22. Liu L, Moody J and Gall A: A quantitative, pooled analysis and systematic review of controlled trials on the impact of electrical stimulation settings and placement on pressure ulcer healing rates in persons with spinal cord injuries. Ostomy Wound Manage 62: 16-34, 2016.

23. Üstün N, Aras M, Ozgur T, Bayraktar HS, Sefil F, Ozden R and Yagiz AE: Thymoquinone attenuates trauma induced spinal cord damage in an animal model. Ulus Travma Acil Cerrahi Derg 20: 328-332, 2014.

24. Foroughi Asl H, Talukdar HA, Kindt AS, Jain RK, Ermel R, Ruusalepp A, Nguyen KD, Dobrin R, Reilly DF, Schunkert H, et al: Expression quantitative trait Loci acting across multiple tissues are enrichedininheritedriskforcoronaryarterydisease.CircCardiovasc Genet 8: 305-315, 2015.

25. Tian DS, Liu JL, Xie MJ, Zhan Y, Qu WS, Yu ZY, Tang ZP, Pan DJ and Wang W: Tamoxifen attenuates inflammatory-mediated damage and improves functional outcome after spinal cord injury in rats. J Neurochem 109: 1658-1667, 2009.

26. Paterniti I, Genovese T, Crisafulli C, Mazzon E, Di Paola R, Galuppo M, Bramanti P and Cuzzocrea S: Treatment with green tea extract attenuates secondary inflammatory response in an experimental model of spinal cord trauma. Naunyn Schmiedebergs Arch Pharmacol 380: 179-192, 2009.

27. Jiang S, Bendjelloul F, Ballerini P, D'Alimonte I, Nargi E, Jiang C, Huang $\mathrm{X}$ and Rathbone MP: Guanosine reduces apoptosis and inflammation associated with restoration of function in rats with acute spinal cord injury. Purinergic Signal 3: 411-421, 2007. 
28. Uchida K, Nakajima H, Watanabe S, Yayama T, Guerrero AR, Inukai T, Hirai T, Sugita D, Johnson WE and Baba H: Apoptosis of neurons and oligodendrocytes in the spinal cord of spinal hyperostotic mouse (twy/twy): Possible pathomechanism of human cervical compressive myelopathy. Eur Spine J 21: 490-497, 2012.

29. Zhang SQ, Wu MF, Gu R, Liu JB, Li Y, Zhu QS and Jiang JL: Senegenin inhibits neuronal apoptosis after spinal cord contusion injury. Neural Regen Res 11: 657-663, 2016.

30. Mizuno A, Miyauchi K, Nishizaki Y, Yamazoe M, Komatsu I, Asano T, Mitsuhashi H, Nishi Y, Niwa K and Daida H: Impact of the augmentation time ratio on direct measurement of central aortic pressure in the presence of coronary artery disease. Hypertens Res 38: 684-689, 2015.

31. Xu GY, Liu S, Hughes MG and McAdoo DJ: Glutamate-induced losses of oligodendrocytes and neurons and activation of caspase-3 in the rat spinal cord. Neuroscience 153: 1034-1047, 2008.

32. Griggs RB, Donahue RR, Morgenweck J, Grace PM, Sutton A, Watkins LR and Taylor BK: Pioglitazone rapidly reduces neuropathic pain through astrocyte and nongenomic PPAR $\gamma$ mechanisms. Pain 156: 469-482, 2015.

33. Paterniti I, Impellizzeri D, Crupi R, Morabito R, Campolo M, Esposito E and Cuzzocrea S: Molecular evidence for the involvement of PPAR- $\delta$ and PPAR- $\gamma$ in anti-inflammatory and neuroprotective activities of palmitoylethanolamide after spinal cord trauma. J Neuroinflammation 10: 20, 2013.

34. Park SW, Yi JH, Miranpuri G, Satriotomo I, Bowen K, Resnick DK and Vemuganti R: Thiazolidinedione class of peroxisome proliferator-activated receptor gamma agonists prevents neuronal damage, motor dysfunction, myelin loss, neuropathic pain, and inflammation after spinal cord injury in adult rats. J Pharmacol Exp Ther 320: 1002-1012, 2007.
35. Pei X, Li X, Chen H, Han Y and Fan Y: Thymoquinone inhibits angiotensin II-induced proliferation and migration of vascular smooth muscle cells through the AMPK/PPAR $\gamma /$ PGC- $1 \alpha$ pathway. DNA Cell Biol 35: 426-433, 2016.

36. Isele NB, Lee HS,Landshamer S,Straube A,Padovan CS,PlesnilaN and Culmsee $\mathrm{C}$ : Bone marrow stromal cells mediate protection through stimulation of PI3-K/Akt and MAPK signaling in neurons. Neurochem Int 50: 243-250, 2007.

37. Zhang P, Zhang L, Zhu L, Chen F, Zhou S, Tian T, Zhang Y, Jiang X, Li X, Zhang C, et al: The change tendency of PI3K/Akt pathway after spinal cord injury. Am J Transl Res 7: 2223-2232, 2015.

38. Felix MS, Bauer S, Darlot F, Muscatelli F, Kastner A, Gauthier P and Matarazzo V: Activation of Akt/FKHR in the medulla oblongata contributes to spontaneous respiratory recovery after incomplete spinal cord injury in adult rats. Neurobiol Dis 69: 93-107, 2014.

39. Liu H, Liu HY, Jiang YN and Li N: Protective effect of thymoquinone improves cardiovascular function, and attenuates oxidative stress, inflammation and apoptosis by mediating the PI3K/Akt pathway in diabetic rats. Mol Med Rep 13: 2836-2842, 2016. 